\title{
Notch signalling in cancer stem cells
}

\author{
Victoria Bolós ${ }^{1}$, Moisés Blanco ${ }^{2}$, Vanessa Medina ${ }^{2,3}$, Guadalupe Aparicio ${ }^{3}$, Silvia Díaz-Prado ${ }^{2,4}$, \\ Enrique Grande ${ }^{1}$
}

1. Medical Oncology Department, Pfizer Spain, Alcobendas, Madrid, Spain

2. Institute for Biomedical Research (INIBIC), A Coruña University Hospital, A Coruña, Spain

3. Oncology Research Unit, A Coruña University Hospital, A Coruña, Spain

4. Medicine DepartmentUniversity of A Coruña, A Coruña, Spain

\begin{abstract}
A new theory about the development of solid tumours is emerging from the idea that solid tumours, like normal adult tissues, contain stem cells (called cancer stem cells) and arise from them. Genetic mutations encoding for proteins involved in critical signalling pathways for stem cells such as BMP, Notch, Hedgehog and Wnt would allow stem cells to undergo uncontrolled proliferation and form tumours. Taking into account that cancer stem cells (CSCs) would represent the real driving force behind tumour growth and that they may be drug resistant, new agents that target the above signalling pathways could be more effective than current anti-solid tumour therapies. In the present paper we will review the molecular basis of the Notch signalling pathway. Additionally, we will pay attention to their role in adult stem cell self-renewal, and cell fate specification and differentiation, and we will also review evidence that supports their implication in cancer.
\end{abstract}

\section{Keywords}

Notch pathway; Cancer stem cells

\section{Introduction}

Notch signalling is a developmental conserved signalling pathway that plays a fundamental role in embryonic development and adulthood. During embryonic development, Notch activation allows definition of the cell fate of groups of embryonic stem cells. The Notch pathway also has a critical role in the self-renewal of adult tissues due to its involvement in the differentiation of non differentiated somatic stem cell populations into specific cell types.

The Notch pathway uses both membrane-bound ligands and receptors. This fact makes fundamental the existence of intercellular contact for the signal transmission.

Notch is a large membrane protein that is expressed in signal-receiving cells and, therefore, it works as a receptor. In mammals, there are four Notch receptors (Notch1-4) with a complex dimeric structure composed of transmembrane monomer bound through non-covalent interactions to a fully extracellular monomer. On the one hand, the intra-cellular face of the transmembrane monomer contains a number of important domains which are involved in the transduction and modulation of the signal. Immediately next to the membrane there is a RAM (RBPjk Associate Molecule) domain followed by a number of motifs name dAnkyrin repeats, which mediates the interaction with the Csl (CBF1 in mammals/Su(H) in Drosophila/Lag-1 in Caenorhabditis elegans) transcription factor. Additionally, there is a transactivation domain (TAD), two nuclear localisation signals (NLS) and a PEST region, which is the site for Notch ubiquitination. 
Therefore, the PEST domain negatively regulates protein stability. On the other hand, the extracellular face of the Notch transmembrane monomer shows a hydrophobic heterodimerisation region which mediates the binding to the Notch extracellular monomer (Fig. 1).

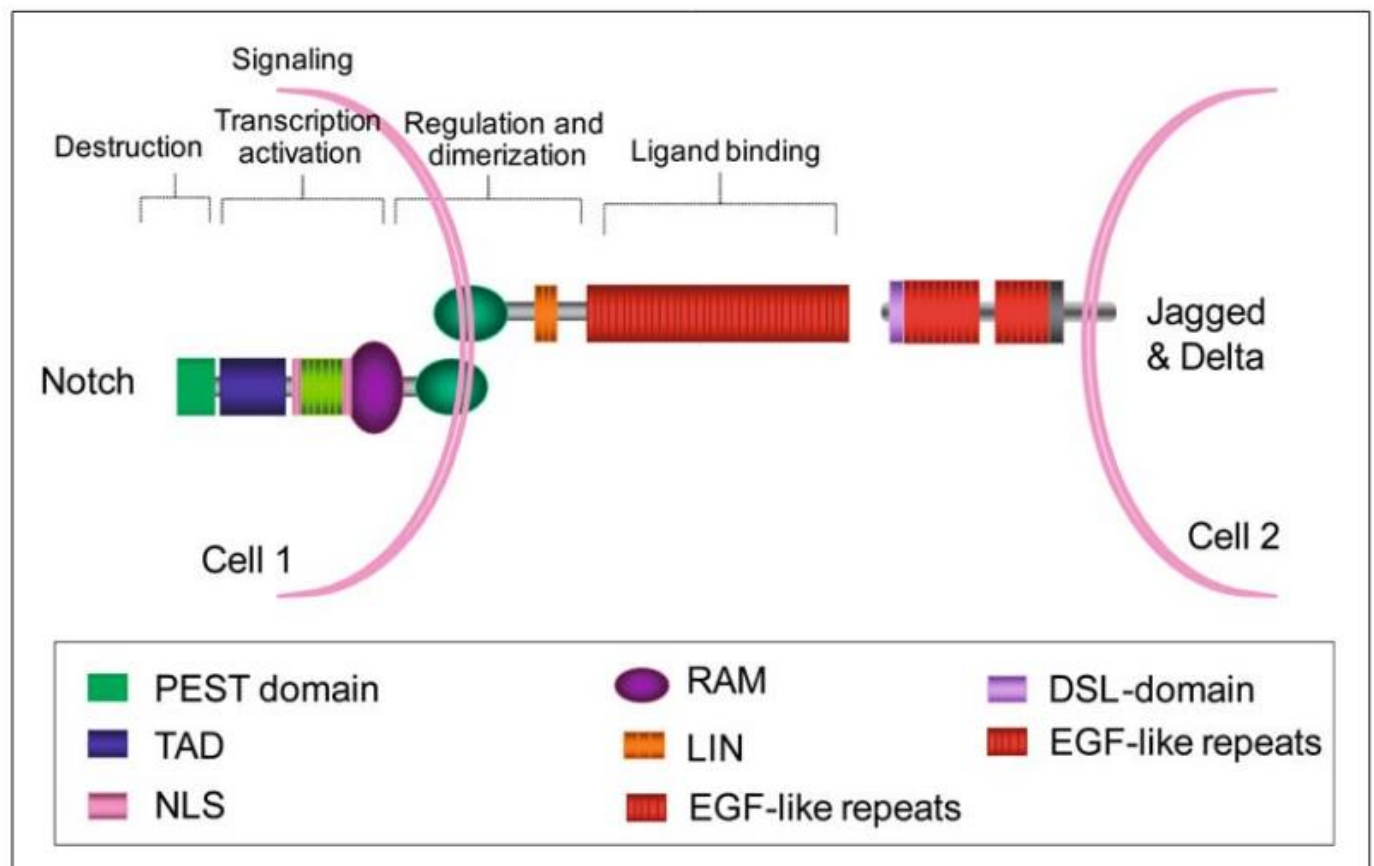

Fig. 1. Notch signalling elements. NOTCH proteins are single-pass transmembrane receptors harbouring alarge extracellular domain involved in ligand binding and a cytoplasmic domain involved in signal transduction. The extracellular domain contains multiple EGF-like portions that are critical for ligand binding. The EGF-like portions are followed by a LIND domain (three cysteine-rich repeats) that prevents signalling in the absence of the signal. The intracellular domain is involved in protein-protein interactions and in the activation of the transcription. In fact, it contains a TAD and two NLS. The intracellular PEST sequence negatively regulates protein stability. Notch ligands are also bound to neighbouring cells. Ligands have an amino-terminal domain termed DSL (for Delta, Serrate and LAG-2 domain), followed by a different number of EGF-like portions. Adapted from [19]

The extracellular Notch monomer is composed, in addition to the heterodimerisation region, of a number of cysteine-rich Lin12 repeats and, in the more distant zone, more than 36 epidermal growth factor (EGF)-like repeats that are critical for the interaction with the Notch ligand(fundamentally 11 and 12 EGF-like repeats).

In mammals there are five conventional ligand molecules: three delta-like ligands (homologous to the delta ligand of Drosophila), D111, D113 and D114, and two jagged ligands (homologous to the serrate ligand of Drosophila), Jag1 and Jag2 (Fig. 1). Both families of ligand are also transmembrane proteins with a shorter intracellular face than Notch receptors. Jagged ligands display a cysteine-rich region followed by less EGF-like repeats than Notch and, finally, a DSL (Delta, Serrate and LAG2) domain. Al-though delta-like ligands contain the entire DSL region, they lack a cysteine-rich region and have less EGF-like re-peats than jagged ligands. DSL regions are functionally important because they are responsible for interacting with the 11 and 12 EGFlike repeats of the Notch receptor. Additionally, the Notch EGF-like repeats $23-25$ can also interact with delta-like ligands but not with the jagged ligands [1]. 
Currently, a new model of development of tumours is emerging and supports the idea that tumours, like normal adult tissues, arise from stem cells. In this model cancer stem cells (CSC) would be the real driving force behind tumour growth. CSCs would retain the differentiation potential giving rise to non-self-renewing differentiated tumour cells that would constitute the bulk of the tumour. It seems that genetic alterations in critical signalling pathways that govern stem cells such as Notch, Wnt, BMP (Bone Morphogenic Protein) and Hedgehog signalling pathways would allow stem cells to become independent of growth signals or resist antigrowth signals and would allow them to undergo uncontrolled proliferation and tumorigenesis[2]. As we will review below, a growing amount of evidence has already shown an association between alterations in the Notch signalling pathway and cancer, supporting the hypothesis mentioned above [3]. In fact, based on published reports that show the relationship of Notch signalling and cancer, clinical trails with Notch signalling inhibitors are underway in cancer patients.

\section{A walk along the Notch pathway}

A number of proteins participate in Notch-mediated signalling, some of them involved in Notch receptor processing [4]. Initially, Notch is synthesised as a large entire molecule that subsequently is cleaved in the Golgi by a furin-like convertase during the export process to the plasmatic membrane. Furthermore, Notch is also glycosylatedin the Golgi. The glycosylation profile seems to be critical for the binding of Notch to a specific ligand. In Drosophilatwo enzymes, O-fut1 (O-fucosyltransferase) and fringe (O-fucosylpeptide $\beta-1,3-\mathrm{N}$ acetylglucosaminyltransferase), are involved in Notch receptors and ligand glycosylation. Invertebrates this process is carried out by the $\mathrm{O}$-fut 1 homologue Pofut1 and by the fringe homologous Lunatic, Manic and Radical Fringe.

The Notch canonical pathway starts when a transmembrane ligand (that belongs to the Deltalike or Jagged family) in a signal-sending cell interacts with the Notch extra-cellular region of a signal-receiving neighbourhood cell[5]. Subsequently, the signal-sending cell, which expresses the ligand, cleaves the Notch extracellular domain that interacts with the ligand in the Notch S1 site and endocytes this ligand-receptor complex. The release of the Notch extracellular domain allows the exposition in the Notch trans-membrane domain of the signal-receiving cell of a site fora new cleavage (the S2 site) by a membrane metalloprotease belonging to the ADAM/TACE (desintegrin and metallopeptidase/tumour necrosis factor aconverting enzyme) family (Adam10 and Adam17 in mammals). As a result of this cleavage, the transmembrane- intracellular segment of Notch is endocyted by the signal- receiving cell, which subsequently undergoes another cleavage in the receptor (in the S3 and S4 sites) by a $\gamma$ - secretase complex (composed presently of 1 and 2 , the catalytic components, and nicastrin, Aph1 and Pen2 in mammals). This cleavage leads to the final release of the cytoplasmatic intracellular active form of Notch, the Notch intracellular domain (NICD)(Fig. 2).

Then, NICD translocates to the nucleus and binds to the transcription factor C-promoter binding factor 1 (Cbf1) that is the Csl transcription factor in mammals. This inter-action converts Cbf1 from a transcriptional repressor into a transcriptional activator of target genes by displacing nu-clear co-repressor and histone deacetylase complexes and recruiting nuclear co-activator proteins such as Mastermind(Fig. 2).

In contrast, Notch signalling is switched off through the binding of Skip (Ski-Interacting Protein) protein to the Cbf1:NICD:Mastermind complex. Skip promotes the recruitment of kinases, which phosphorylate TAD and PEST domains of NICD. These phosphorylated sites are then recognised by specific ubiquitin ligases that trigger NICD destruction (Fig. 1). Finally, Cbf1 returns to its initial state as a transcriptional repressor of target genes [1]. 


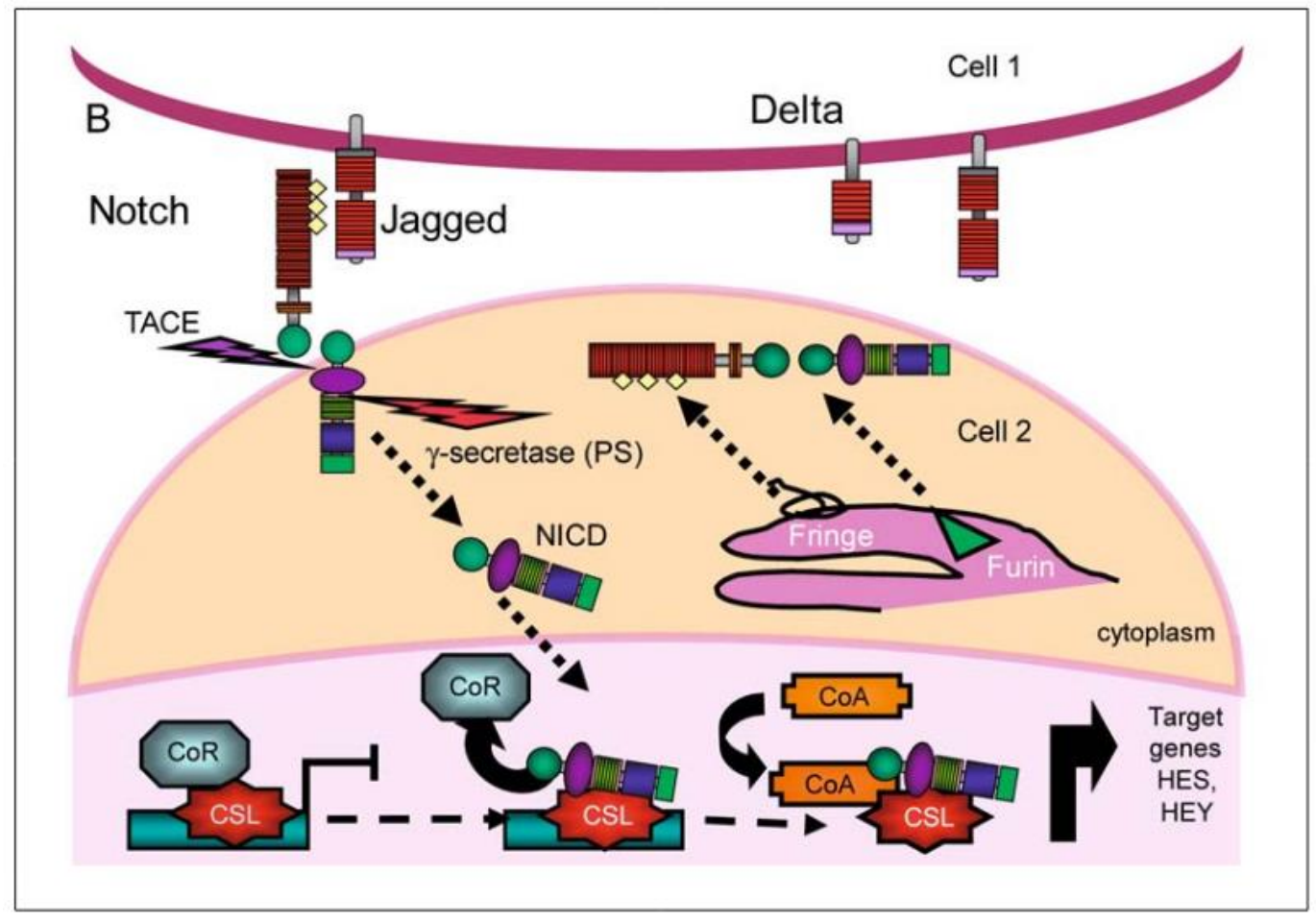

Fig. 2. Notch signalling pathway. Notch receptors are synthesised as single precursor proteins that are cleaved during transport to the cell surface, where they are expressed as heterodimers. Following the binding of the ligand, placed in the surface of a neighbouring cell, NOTCH is activated by two consecutive proteolytic cleavages that release its intracellular domain (NICD). The first proteolytic cleavage is mediated by the metalloprotease TACE, which cleaves the receptor on the extracellular side, near the transmembrane domain. The second cleavage occurs within the transmembrane domain and is mediated by a gamma-secretase activity whose key component is presenilin. This final cleavage liberates the NICD, which subsequently translocates to the nucleus where it binds to the transcription factor CBF1. This interaction converts $\mathrm{CBF} 1$ from a transcriptional re-pressor into a transcriptional activator by displacing nuclear co-repressor proteins (CoR) and through the recruitment of nuclear co-activator proteins (CoA). Adapted from [19]

Although most of the Notch cellular responses occur as a result of the activation of the canonical Notch pathway described above, there are other proteins that may also act as Notch ligands and trigger Notch activation. This non-canonical Notch pathway involves other transmembrane proteins with EGF-like repeats such as Dner, F3/Contactin1 and NB-3/Contactin 6. However, these Notch ligands bind Notch receptors with less affinity than the convention-al Notch ligands (delta and Jagged) due to the lack of the DSL region [6].

Finally, Notch signalling may involve a number of other proteins such as CCN proteins, which are secreted to the extracellular environment where they may act as regulators of the Notch signal [7]. 


\section{Notch signalling and trafficking: ligand and receptorendocytosis}

In several observations in Drosophila, it has been seen that the mutants with defects in the mechanisms of endocytosis and vesicular trafficking also display defects in Notch signalling. These findings suggest that endocytosis and cellular trafficking are necessary and are linked with Notch signalling [1].

On the one hand, once ligand-receptor interaction takes place, the first step for triggering Notch endocytosis/internalisation seems to be its mono-ubiquitination. Notch internalisation leads to the proteolytic cleavage of the receptor by the $\gamma$ - secretase complex and the subsequent NICD release and translocation to the nucleus to regulate the expression of target genes. On the other hand, Notch ubiquitination and endocytosis, without previous interaction of Notch with a specific ligand, may give rise to two different responses: Notch degradation or Notch activation in a ligand-independent manner [8].

Three ubiquitin ligases that are able to trigger Notch endocytosis have been identified in Drosophila: Deltex(Dx), Nedd4 and Suppressor of Deltex (Su(Dx)). Deltex labels with ubiquitins the ankyrin-rich domain of Notch. Nedd4 and $\mathrm{Su}(\mathrm{Dx})$ label the HECT (homologous to E6APcarboxyl terminus) domain of Notch with ubiquitins. Al-though the role of $\mathrm{Dx}, \mathrm{Su}(\mathrm{Dx})$ and Nedd4 is unclear, it is likely that they may regulate Notch signalling [8]. Since Dx seems to have a positive effect on Notch signalling activity, it seems that the Dx-mediated endocytosis of Notch avoids Notch degradation after its activation in a ligand-in-dependent manner. However, both Nedd4 and $\mathrm{Su}(\mathrm{Dx})$ have a negative effect on Notch signalling, leading the Notch-containing endocytic vesicles toward degradation.

Furthermore, evidence suggests that ubiquitination and endocytosis of Notch ligands are also necessary for triggering Notch signalling. Ligand endocytosis after ligand-receptor interaction promotes the separation of Notch extracellular fragments (also endocyted by the ligandexpressing cells) and exposes specific sites in the transmembrane region of the receptor for cleavage by ADAM proteases. Additionally, it has also been suggested that ligand endocytosis leads to its post-translational modification. Therefore, it seems that after ligand endocytosis and modification, the modified ligand is competent to activate the signaling through its interaction with Notch and a subsequent ligand-receptor endocytosis [8]. Notch modification by the Pofut1 fucosyltransferase also seems to be important for its endocytosis-dependent signalling [9].

\section{Notch in action: normal functions, target genesand mechanisms of action}

Many of the cellular responses carried out by the Notch pathway have been discovered through the analysis of the phenotype derived from mutations in Drosophila Notch genes. These mutations mainly affect the development of the nervous and sensory organs.

The main effectors of Notch activation that promote Notch-mediated cellular responses are transcription factors belonging to the basic helix-loop-helix (bHLH) family. In Drosophila, a gene known as Hairy and a gene cluster called Enhancer of split complex (E(spl)-C) are effectors of Notch activation taking part in the development of nervous components as well as in muscle and visceral development. In mammals the Hes (Hairy Enhancer of split) family, composed of seven genes (Hes1-7), is homologous to the Drosophila Hairy and E(spl)-C.Hes1plays an important role in nervous system and sensory organ development, as well as in the development of pancreas and other endocrine cells [10]. Hes1 and Hes7 are also involved in somitogenesis and, therefore, in the development of the body pattern [11]. Hey, also known as Hrt, Hesr, Herpor Chf, is a subfamily of Hairy and Enhancer of split related genes that is also involved in Notch response in vertebrates. This subfamily of genes is critical for the development of the cardiovascular system [10]. 
Notch is involved in cell fate decisions in embryonic and adult stem cells and progenitors. Notch is also involved in terminal differentiation events in specific tissues. Additionally, Notch promotes the development of new cell types at the boundaries of two distinct cell populations. Thus, there are several processes where Notch is involved. These processes affect the control of melanocyte generation and epidermal development [12, 13], cardiac and vascular sys-tem development [14-16], the development of haematopoietic cell lineage, gastric mucosa renewal and nervous sys-tem development [17]. Notch signalling has also been shown to be essential for proper prostate development in male mammals [18].

Besides bHLH transcription factors, other genes have been identified as Notch targets and some of them are tissue specific. Among these genes are the protooncogene c-Myc, Skip2 and Deltex1 genes (both of which encode for proteins with ubiquitin ligase activity) and genes that encode for cell cycle regulators such as p27KIP1 and cyclin D1[19].

Notch is able to allocate the suitable fates to different cells. In this case Notch signal must be directed. Therefore, one cell must assume the role of signal-sending cell ex-pressing high levels of Notch ligands, whereas another neighbour cell may play the role of signal-receiving cell, and therefore this cell must express high levels of Notch receptors. However, neighbourhood cells usually express both ligands and receptors at the same level, making it impossible to distinguish the cell that sends the signal and the cell that receives it. There are several models to explain how the directionality in the signalling is achieved: the lateral inhibition model as well as asymmetric cell division and modulation of the activity of the ligands and the receptors [20]. In addition, it has also been shown that lateral induction functions as a system for control of the directionality in Delta-Notch signalling [21-23]. In this way, Serrate1 is regulated by Notch-mediated lateral induction in supporting cells during development of the inner ear in vertebrates [24]. Additionally, a theoretical model has also been developed where-by it was demonstrated that lateral induction is involved in the control of epidermal stem cell niches [25].

The lateral inhibition is based on the ability of the Notch pathway to amplify small differences in the capacity of Notch signal transmission between different cells. These models suggest that small differences in the activity of the Notch pathway in distinct cells may have an influence on the level of expression of their ligands and/or receptors, inducing autoregulation loops that generate a "snowball" effect. In the lateral inhibition model one cell gradually loses the expression of Notch receptors until eventually it carries only Notch ligands (this cell becomes the signalsending cell), while the other cell gradually loses the expression of Notch ligands until it eventually carries only receptors (this other cell becomes the signal-receiving cell). The gonadal development in $\mathrm{C}$. elegans is a good example of lateral inhibition. The receptor activates its own expression and inhibits the expression of the ligand in the signal-receiving cell.

The Notch signal also acquires directionality by asymmetric cell division. In this case, the direction of Notch signalling is determined by the asymmetrical distribution during cell division of a number of factors that influence Notch activity in daughter cells. The mother cell has located these factors at one of its poles instead of displaying a homogeneous distribution of such factors. One of the factors capable of modifying the Notch signal is Numb, a protein that binds Notch directly to avoid Notch activation. Therefore, although these two cells are competent for Notch signalling since both express ligands and receptors, only the cell without Numb protein expression will be able to receive the signal via Notch, while the other cell with Numb will send the signal.

Finally, another mechanism to achieve directionality of Notch signalling is controlling the ligand and receptor activities through post-translational modifications. As has previously been depicted, the ubiquitin-dependent endocytosis of both Notch receptor and ligands has important consequences for their activity and, therefore, for the modulation of Notch pathway activity. In mammals, two ubiquitin ligases called Mindbomb and Neuralized are capable of ubiquitinating the Notch ligands and thus triggering their endocytosis. As a result of their action, Mind-bomb and Neuralized enhance the ligand capacity to activate the Notch signalling in the surrounding cells. Additionally, Notch activity can be controlled through glycosylation of its EGF-like repeats by glycosyl-transferases such as fringe in Drosophila and its counterparts in vertebrates. Distinct 
spatial patterns of expression of these proteins during embryonic development can generate the directionality of the Notch signalling, giving rise to different cell groups in the organism [20].

\section{The two faces of Notch in cancer}

Deregulated expression of wild-type Notch receptors, ligands and target genes is observed in a growing number of solid and haematological tumours. Mutations in Notch receptors have also shown tumorigenic properties in preclinical models [3].

However, evidence shows that the Notch signaling pathway has apparently opposite functions in tumour development, where it may act either as an oncogene or as a tumour suppressor, depending on the cell context. In fact, it seems that the result of alteration in Notch signalling is de-pendent on its normal function in a given tissue. In this set-ting, Notch may act as an oncogene in those tissues where it is involved in stem cell self-renewal or in cell fate decisions. In contrast, Notch signalling may have a tumour suppressor role in those tissues in which Notch promotes terminal differentiation events (reviewed in [3]). Therefore, with the possible exception of keratinocyte-derived tumours, where Notch would have a tumour suppressor role, Notch signalling inhibition may be an effective strategy for the treatment of certain solid and haematological tumours (reviewed in [26-28]).

\section{Oncogenic role of Notch}

\section{Breast cancer}

Because mammary glands undergo most of their development after birth and show great plasticity during a woman's life, adult stem cells play a pivotal role in the biology of the mammary gland [29]. Notch signalling is involved in mammary stem cell self-renewal [30, 31]. In fact, induction of Notch signalling promotes self-renewal of mammary stem cells and branching morphogenesis and these effects are blocked by an anti-Notch antibody or a gamma-secretase inhibitor [30, 31].

Recently, it has been suggested that residual breast tumours after conventional chemotherapy are enriched with CSCs with increased self-renewal capability due to the alteration of developmental conserved signalling pathways such as Notch [32]. Accordingly, in preclinical models Notch signalling protects breast tumours from drug-induced apoptosis [33].

Activation of Notch signalling occurs in approximately $40 \%$ of human breast tumours. In fact, NOTCH1 and NOTCH2 expression have been associated with clinical outcome. It seems that NOTCH1 may have tumour-promoting functions, whereas NOTCH2 could play a tumoursuppressive role [34]. Parr and co-workers showed that NOTCH1 expression was low in welldifferentiated breast tumours and was increased in poorly differentiated breast tumours, whereas NOTCH2 expression was high in well-differentiated breast tumours and reduced in poorly differentiated breast tumours [34]. These authors suggested that high NOTCH1 levels may be associated with a poor prognosis while high NOTCH2 levels may be associated with a higher overall survival [34]. Further evidence showed that high JAG1 or N1 levels in breast cancer were associated with poor overall survival. Furthermore, a synergistic effect of high levels of coexpression of JAG1 and NOTCH1 on overall survival has also been shown [35].

Additionally, the loss of negative regulators of Notch signalling has been shown in breast cancer. In fact, NUMB-mediated negative control of Notch signalling is lost in $50 \%$ of human breast tumours due to specific NUMB ubiquitination and proteosomal degradation [36]. Furthermore, NUMB-defective breast tumours display poor prognosis [37]. Recently, Colaluca and co-worker shave shown that loss of NUMB protein in primary breast tumour cells causes an increase in Notch signalling, a de-crease in p53 levels and an increase in cell chemoresistance[37]. 
Additionally, it has been suggested that NOTCH1 may mediate the tumorigenic effect of oncogenic RAS[38].Currently, it is well established that gain of function mutations in RAS occurs in early breast cancer and plays a central role in breast tumorigenesis. Weijzen and colleagues showed that NOTCH1 was highly expressed in all RAS-positive breast cancer samples. Downregulation of NOTCH1 expression in RAS-transformed human breast cells led to a significant decrease in their proliferation. Additionally, the inhibition of RAS signalling also led to the blockade of NOTCH1 activation. Furthermore, the expression of oncogenic RAS also led to higher levels of proteins involved in NOTCH activation [38].

A role for Notch signalling in the different breast cancer molecular subtypes has been suggested based on pre-clinical data and Notch signalling component expression in samples from breast cancer patients. Recently, Rizzo and co-workers showed cross-talk between Notch signalling and oestrogen receptor (ER) signalling in ER-positive breast cancer models, while oestradiol inhibited Notch activation in breast cancer cell lines with ER protein levels. However, anti-oestrogen therapy with tamoxifen blocked this inhibitory effect and reactivated Notch activity in these cells. Additionally, Notch inhibition enhanced the anti-tumour effects of tamoxifen in vivo over ER-positive breast cancer models. Finally, these authors suggested that Notch signalling could represent another potential therapeutic tar-get in ER-positive breast cancer (the most common breast cancer molecular subtype). They also showed preclinical data that supported that the combinations of anti-oestrogen drugs and Notch inhibitors may be more effective than an-tioestrogen drugs in monotherapy in this breast cancer molecular subtype [39]. Additionally, the same authors, in another recent article, also showed how ERBB2 over expression suppressed NOTCH1 activity in ERBB2-positivebreast cancer models and how this effect could be reversed by trastuzumab (Trastuzumab is an anti-HER2 inhibitor that is consider the first choice of treatment for ERBB2-positive breast tumors). Notch inhibition also showed an anti-tumour effect in ERBB2-positive breast cancer models that were trastuzumab resistant. These results suggested that Notch might play a role in the resistance to trastuzumab in the ERBB2-positive breast cancer molecular subtype. This resistance may be prevented or reversed by the concomitant or subsequent inhibition of the Notch pathway[40]. Finally, a clearer role for Notch signalling in triple negative breast cancer has also been shown in pre-clinical models. These data suggest that therapeutic targeting of this pathway may be explored in breast cancer patients with this molecular subtype [41].

Based on the above evidence that supports a role for the Notch signalling pathway in breast cancer molecular sub-types, two phase I/II clinical trials with the gamma-secretase inhibitor MK0752 (Merck) in breast cancer patients are underway. These trials will determine the safety/tolerability and efficacy of the MK-0752 inhibitor in monotherapy or combined with docetaxel in locally advanced or metastatic breast cancer patients [42, 43]. In the former tri-al, continuous dosing of MK-0752 at $450 \mathrm{mg}$ daily in patients with breast cancer is associated with significant toxicity, predominantly fatigue [42]. Currently, an intermittent dosing schedule is being explored. In the latter trial, developed by the Stem Cell Clinical Consortium, tumour samples will be taken from some patients who have tumours that can be easily biopsied. The samples will be used to test if "breast CSCs" are killed by the combination of docetaxel and MK-0752 [43].

\section{Gastrointestinal tumours}

The epithelium of the small intestine is organised into finger-like villi and adjacent invaginations called crypts of Lieberkühn. The colon lacks villi and has a flat surface epithelium. In the small intestine, the crypt compartment contains the stem cells and the progenitors, whereas the villus compartment is made up entirely of differentiated cells (re-viewed by [44]). The stem cells of the bottom of the crypt give rise to the transit amplifying cells, which divide robustly before terminal differentiation into one of the four principle cell types: adsorptive enterocytes, mucus-secreting goblet cells, hormone-secreting enteroendocrine cells, and lysozyme- and cryptidin-producing Paneth cells [44].

The self-renewal of the intestine is regulated by highly evolutionary conserved signalling pathways, which include the BMP, Hh, Notch and Wnt [45]. 
Multiple proteins belonging to the Notch signalling pathway are expressed in the intestinal crypts and several Notch target genes are involved in the control of the intestine self-renewal such as Hes1 and Math1 (the latter gene, a target of Hes1-mediated gene repression) (reviewed in[44]). The Notch signalling pathway is required to establish the fate of differentiating cells exiting from the transit-amplifying compartment. Notch has also been shown to have an essential role within the crypt compartment to maintain crypt progenitors in an undifferentiated, proliferative state (reviewed in [44]).

Cross-talk between Wnt and Notch intracellular path-ways has also been suggested by van Es and coworkers. Notch might be acting downstream on Wnt in the intestine self-renewal and in the promotion of proliferation in adenomas and adenocarcinomas of the intestine [46].

Disruption of Notch signalling in colon cancer cell lines by gamma-secretase inhibitors induced apoptosis $[47,48]$. Furthermore, it has been shown that the pre-treatment of these cell lines with gamma-secretase inhibitors enhances oxaliplatin effects [47] and also enhances the action of taxanes [48].

Additional evidence indicates that the Notch signalling pathway may be associated with chemotherapy resistance in gastric tumours [48, 49]. Oxaliplatin-resistant gastric cancer cell lines showed increased levels of VEGF and the NOTCH ligands JAG1 and DLL4, associated with a higher angiogenic rate [49].

\section{Haematological tumours: T-cell lymphoblastic leukaemia/lymphoma}

Notch signalling has an important role in haematopoiesisas a mediator of cell fate determination and in the self-renewal of haematopoietic stem cells [50].

The Notch signalling pathway also plays a central role in T-cell acute lymphoblastic leukaemia/lymphoma (T-ALL) pathogenesis. Less than $1 \%$ of T-ALL show a chromosome translocation [42, 47] that leads to the expression of constitutively active Notch1 (N1ICD). Additionally, more than $50 \%$ of human T-ALLs without specific [42, 47] chromosome translocation show activating mutations inNotch-1([51], reviewed in [52]). Based on these findings, a safety/efficacy phase I clinical trial with the gamma-secretase inhibitor MK-0752 in patients with refractory T-ALL was developed by Merck [53].

\section{Tumour suppressor role}

\section{Keratinocyte-derived carcinoma}

Notch1-mediated signalling in the epidermis promotes the commitment of stem cells toward transit populations of cells that are still actively proliferating, although only for a limited time until they differentiate [54]. Thus, the function of Notch1 in this tissue is to induce terminal differentiation processes. Notch1 in primary mouse keratinocytes has shown a tumour suppressive role. Notch1 promotes the withdraw-al of proliferating primary keratinocytes from the cell cycle and their entrance into differentiation $[55,56]$. Conditional deletion of the Notch1gene in the epidermis of mouse models results in epidermal hyperplasia and development of spontaneous basal cell carcinoma-like tumours and facilitation of chemical-induced skin carcinogenesis (reviewed in [3]).

The tumour suppressor effect of Notch1 in murine skin appears to be mediated by the repression of signalling pathways implicated in self-renewal of skin stem cells such as $\mathrm{Hh}$ and Wnt [56-58]. 
According to the tumour suppressor role of Notch in mouse skin, human basal cell carcinomas show reduced Notch1, Notch2 and JAG1 expression [57]. Furthermore, basal cell carcinoma is frequently associated with higher Hh signalling. Consistent with these data, Notch1deletionin mouse epidermis leads to aberrant expression of Gli2, a downstream component of the $\mathrm{Hh}$ cascade. Therefore, loss of Notch1 in human epidermis may be contributing, as well as aberrant Hh signalling, to the development of basal cell carcinomas [3].

Epidermal growth factor receptor (EGFR) signalling is associated with skin cancer. Accordingly with the tumour suppressor role of Notch1 in the skin, EGFR has been shown as a negative regulator of Notch1transcription in keratinocytes [59].

Increased Wnt signalling is also associated with the maintenance of stem cell compartments in the skin and with keratinocyte-derived malignancies. Wnt signalling, as well as Hh signalling, is suppressed by Notch-1 activation and is elevated in keratinocytes and tumours as a consequence of loss of Notch1 function [56].

\section{Conclusions}

The hypothesis that cancers may arise from CSCs, which share the self-renewal feature of normal stem cells, has changed the perspective on new approaches to treating the disease such as CSC target therapies.

Developmental signalling pathways such as Notch, BMPs, Wnt and Hh play critical roles in controlling adult stem cells in homeostatic tissues. Alterations in the components of these pathways have also been associated with cancer. In fact, the Notch signalling pathway is a highly evolutionary conserved signalling pathway involved in proliferation and differentiation of embryonic and adult stem cells and also seems to be involved in cancer development. If we take into account the above information, it seems reasonable to consider these signalling pathway components as good targets for the development of new anticancer drugs.

Currently, five clinical trials with the gamma secretase inhibitor MK-0752 from Merck are underway in cancer patients (breast cancer, central nervous system cancer and leukaemia and lymphoma patients). Furthermore, other pharmaceutical companies are also working on the development of drugs against the Notch pathway. They are not only focusing on obtaining new gamma secretase inhibitors capable of simultaneously blocking all Notch isoforms, they are also looking for monoclonal antibodies against specific Notch receptors and ligands to reduce the treatment-associated toxicities.

Further, clinical trials with these new anti-Notch agents in combination with current anticancer therapies are necessary to provide proof of concept of their activity over CSC in a specific tumour type.

Acknowledgements. MBC is supported by Instituto de Salud CarlosIII (Spanish Goverment) through its research support programme. SDP has a contract with the Isidro Parga Pondal programme from Xunta de Galicia (Spain). VMV and GAG are supported by A Coruña University Hospital Foundation.

Conflict of interest. Enrique Grande and Victoria Bolós are employed by and own stock in Pfizer. They were involved in the conception and writing of the manuscript and gave approval for its final version. 


\section{References}

1. Fiúza UM, Arias AM (2007) Cell and molecular biology of Notch. J Endocrinol 194:459-474

2. Ailles LE, Weissman IL (2007) Cancer stem cells in solid tumors. Curr Opin Biotechnol 18:460-466

3. Bolós V, Grego-Bessa J, de la Pompa JL (2007) Notch signaling in development and cancer. Endocr Rev 28:339-363

4. Schweisguth F (2004) Regulation of notch signaling activity. Curr Biol 14:R129-138

5. Ehebauer M, Hayward P, Martinez-Arias (2006) Notch signaling pathway. Sci STKE 364:cm7

6. Katoh M, Katoh M (2007) Notch signaling in gastrointestinal tract. Int J Oncol 30:247-251

7. Katsube K, Sakamoto K (2005) Notch in vertebrates - molecular aspects of the signal. Int J Dev Biol 49:369-374

8. Le Borgne R, Bardin A, Schweisguth F (2005) The roles of receptor and ligand endocytosis in regulating Notch signaling. Development 132:1751-1762

9. Vodovar N, Schweisguth F (2008) Functions of Ofucosyltransferase in Notch trafficking and signaling: towards the end of a controversy? J Biol 7:7

10. Fischer A, Gessler M (2007) Delta-Notch - and then? Protein interactions and proposed modes of repression by Hes and Hey bHLH factors. Nucleic Acids Res 35:4583-4596

11. Kageyama R, Masamizu Y, Niwa Y (2007) Oscillator mechanism of Notch pathway in the segmentation clock. Dev Dyn 236:1403-1409

12. Schouwey K, Beermann F (2008) The Notch pathway: hair graying and pigment cell homeostasis. Histol Histopathol 23:609-619

13. Watt FM, Estrach S, Ambler CA (2008) Epidermal Notch signalling: differentiation, cancer and adhesion. Curr Opin Cell Biol 20:171-179

14. Niessen K, Karsan A (2007) Notch signaling in the developing cardiovascular system. Am J Physiol Cell Physiol 293:C1-11

15. Siekmann AF, Covassin L, Lawson ND (2008) Modulation of VEGF signalling output by the Notch pathway. Bioessays 30:303-313

16. Gridley T (2007) Notch signaling in vascular development and physiology. Development 134: 2709-2718

17. Chiba S (2006) Notch signaling in stem cell systems. Stem Cells 24:2437-2447

18. Leong KG, Gao WQ (2008) The Notch pathway in prostate development and cancer. Differentiation 76 : 699-716

19. Koch U, Radtke F (2007) Notch and cancer: a double-edged sword. Cell Mol Life Sci 64:2746-2762

20. Lai EC (2004) Notch signaling: control of cell communication and cell fate. Development 131:965-973

21. Lewis J (1998) Notch signalling and the control of cell fate choices in vertebrates. Semin Cell Dev Biol 9:583-589

22. de Celis JF, Bray S (1997) Feed-back mechanisms affecting Notch activation at the dorsoventral boundary in the Drosophila wing. Development 124:3241-3251

23. Huppert SS, Jacobsen TL, Muskavitch MA (1997) Feedback regulation is central to Delta-Notch signalling required for Drosophila wing vein morphogenesis. Development 124:3283-3291

24. Eddison M, Le Roux I, Lewis J (2000) Notch signaling in the development of the inner ear: lessons from Drosophila. Proc Natl Acad Sci U S A 97:11692-11699

25. Savill NJ, Sherratt JA (2003) Control of epidermal stem cell clusters by Notch-mediated lateral induction. Dev Biol 258:141-153

26. Nickoloff BJ, Osborne BA, Miele L (2003) Notch signaling as a therapeutic target in cancer: a new approach to the development of cell fate modifying agents. Oncogene 22: 6598-6608

27. Radtke F, Raj K (2003) The role of Notch in tumorigenesis: oncogene or tumour suppressor? Nat Rev Cancer 3:756-767

28. Aster JC (2005) Deregulated NOTCH signaling in acute T-cell lymphoblastic leukemia/lymphoma: new insights, questions, and opportunities. Int J Hematol 82:295-230

29. Charafe-Jauffret E, Monville F, Ginestier C et al (2008) Cancer stem cells in breast: current opinion and future challenges. Pathobiology 75:75-84

30. Dontu G, Al-Hajj M, Abdallah WM et al (2003) Stem cells in normal breast development and breast cancer. Cell Prolif 36[Suppl 1]:59-72

31. Dontu G, Jackson KW, McNicholas E et al (2004) Role of Notch signaling in cell-fate determination of human mammary stem/progenitor cells. Breast Cancer Res 6:R605-615

32. Chang JC, Li X, Wong H et al (2007) Therapeutic resistance and tumor-initiation: Molecular pathways involved in breast cancer stem cell self-renewal. J Clin Oncol ASCO Annual Meeting Proceedings (PostMeeting Edition) 25[Suppl 18]:528

33. Stylianou S, Clarke RB, Brennan K (2006) Aberrant activation of notch signaling in human breast cancer. Cancer Res 66:1517-1525

34. Parr C, Watkins G, Jiang WG (2004) The possible correlation of Notch-1 and Notch-2 with clinical outcome and tumour clinicopathological parameters in human breast cancer. Int J Mol Med 14:779-786

35. Reedijk M, Odorcic S, Chang L et al (2005) High-level coexpression of JAG1 and NOTCH1 is observed in human breast cancer and is associated with poor overall survival. Cancer Res 65:8530-8537 
36. Pece S, Serresi M, Santolini E et al (2004) Loss of negative regulation by Numb over Notch is relevant to human breast carcinogenesis. J Cell Biol 167:215-221

37. Colaluca IN, Tosoni D, Nuciforo P et al (2008) NUMB controls p53 tumour suppressor activity. Nature $451: 76-80$

38. Weijzen S, Rizzo P, Braid M et al (2002) Activation of Notch-1 signaling maintains the neoplastic phenotype in human Ras-transformed cells. Nat Med 8:979-986

39. Rizzo P, Miao H, D'Souza G et al (2008) Cross-talk between notch and the estrogen receptor in breast cancer suggests novel therapeutic approaches. Cancer Res 68:5226-5235

40. Osipo C, Patel P, Rizzo P et al (2008) ErbB-2 inhibition activates Notch-1 and sensitizes breast cancer cells to a gamma-secretase inhibitor. Oncogene 27:5019-5032

41. Lee CW, Raskett CM, Prudovsky I, Altieri DC (2008) Molecular dependence of estrogen receptornegative breast cancer on a notch-survivin signaling axis. Cancer Res 68:5273-5281

42. Krop IE, Kosh M, Fearen I et al (2006) Phase I pharmacokinetic (PK), and pharmacodynamics (PD) trial of the novel oral Notch inhibitor MK-0752 in patients (pts) with advanced breast cancer (BC) and other solid tumors. ASCO Annual Meeting. Abstract No: 1057443. http://clinicaltrials.gov/ct2/show/NCT00645333?intr=\%22MK0752\%22\& rank=2)

44. van Es JH, Clevers H (2005) Notch and Wnt inhibitors as potential new drugs for intestinal neoplastic disease. Trends Mol Med 11:496-502

45. Sancho E, Batlle E, Clevers H (2004) Signaling pathways in intestinal development and cancer. Annu Rev Cell Dev Biol 20:695-723

46. van Es JH, van Gijn ME, Riccio O et al (2005) Notch/gamma-secretase inhibition turns proliferative cells in intestinal crypts and adenomas into goblet cells. Nature 435:959-963

47. Meng RD, Qin L, Shelton CC et al (2008) Use of the Notch signaling pathway to predict disease progression and distant recurrence-free survival in early stage colon cancer. ASCO Annual Meeting. Abstract No: 14500

48. Akiyoshi T, Nakamura M, Yanai K et al (2008) Gamma-secretase inhibitors enhance taxane induced mitotic arrest and apoptosis in colon cancer cells. Gastroenterology 134: 131-144

49. Lim SJ, Fan F, Dallas NA et al (2008) Resistance to oxaliplatin in human intestinal-type gastric cancer cells demonstrates increased migration, invasion and expression of angiogenic factors. ASCO Gastrointestinal Cancer Symposium. Abstract No: 91

50. Duncan AW, Rattis FM, DiMascio LN et al (2005) Integration of Notch and Wnt signaling in hematopoietic stem cell maintenance. Nat Immunol 6:314-322

51. Weng AP, Ferrando AA, Lee W et al (2004) Activating mutations of NOTCH1 in human T cell acute lymphoblastic leukemia. Science 306:269-271

52. Aster JC (2005) Deregulated NOTCH signaling in acute T-cell lymphoblastic leukemia/lymphoma: new insights, questions, and opportunities. Int J Hematol 82:295-301

53. Deangelo DJ, Stone RM, Silverman LB et al (2006) A phase I clinical trial of the notch inhibitor MK0752 in patients with T-cell acute lymphoblastic leukemia/lymphoma (T-ALL) and other leukemias. ASCO Annual Meeting. Abstract No: 6585

54. Lowell S, Jones P, Le Roux I et al (2000) Stimulation of human epidermal differentiation by delta-notch signalling at the boundaries of stem-cell clusters. Curr Biol 10:491-500

55. Rangarajan A, Talora C, Okuyama R et al (2001) Notch signaling is a direct determinant of keratinocyte growth arrest and entry into differentiation. EMBO J 20:3427-3436

56. Nicolas M, Wolfer A, Raj K et al (2003) Notch1 functions as a tumor suppressor in mouse skin. Nat Genet 33:416-421

57. Thelu J, Rossio P, Favier B (2002) Notch signalling is linked to epidermal cell differentiation level in basal cell carcinoma, psoriasis and wound healing. BMC Dermatol 2:7

58. Devgan V, Mammucari C, Millar SE et al (2005) p21WAF1/Cip1 is a negative transcriptional regulator of Wnt4 expression downstream of Notch1 activation. Genes Dev 19:1485-1495

59. Kolev V, Mandinova A, Guinea-Viniegra J et al (2008) EGFR signalling as a negative regulator of Notch1 gene transcription and function in proliferating keratinocytes and cancer. Nat Cell Biol 10:902911 